\title{
Retraction
}

\section{Retracted: Extracting the QCD Cutoff Parameter Using the Bernstein Polynomials and the Truncated Moments}

\author{
Advances in High Energy Physics \\ Received 8 March 2015; Accepted 8 March 2015 \\ Copyright (C) 2015 Advances in High Energy Physics. This is an open access article distributed under the Creative Commons \\ Attribution License, which permits unrestricted use, distribution, and reproduction in any medium, provided the original work is \\ properly cited. The publication of this article was funded by SCOAP ${ }^{3}$.
}

The paper titled "Extracting the QCD Cutoff Parameter Using the Bernstein Polynomials and the Truncated Moments" [1], published in Advances in High Energy Physics, has been retracted as it was found to contain a substantial amount of material from following published articles: "Evolution Equations for Truncated Moments of the Parton Distributions," Phys. Lett. B644 (2007) 284-287; [hep-ph 0610282] and "Truncated Mellin Moments: Useful Relations and Implications for the Spin Structure Function \$g_2\$," Acta Phys. Polon. B42 (2011) 1231-1246; [arXiv: 1106.3753].

\section{References}

[1] A. Mirjalili, M. M. Yazdanpanah, and Z. Moradi, "Extracting the QCD cutoff parameter using the bernstein polynomials and the truncated moments," Advances in High Energy Physics, vol. 2014, Article ID 304369, 7 pages, 2014. 

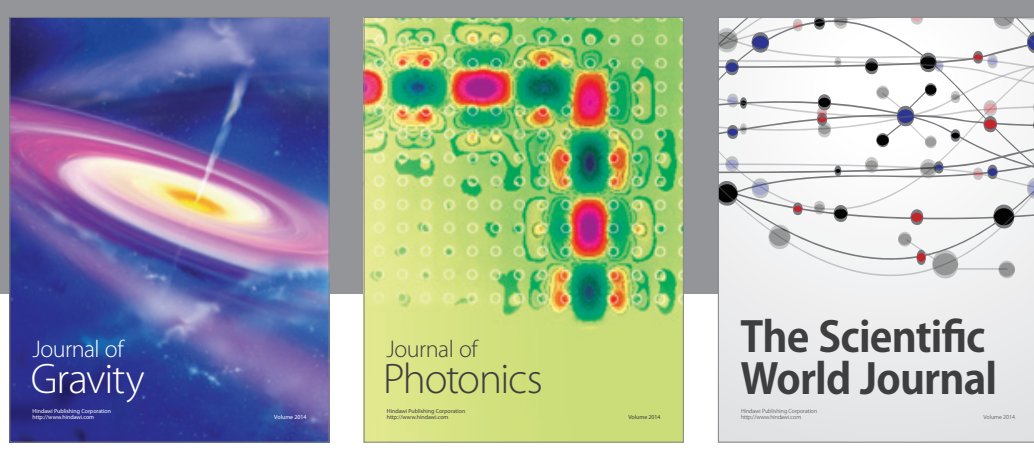

The Scientific World Journal
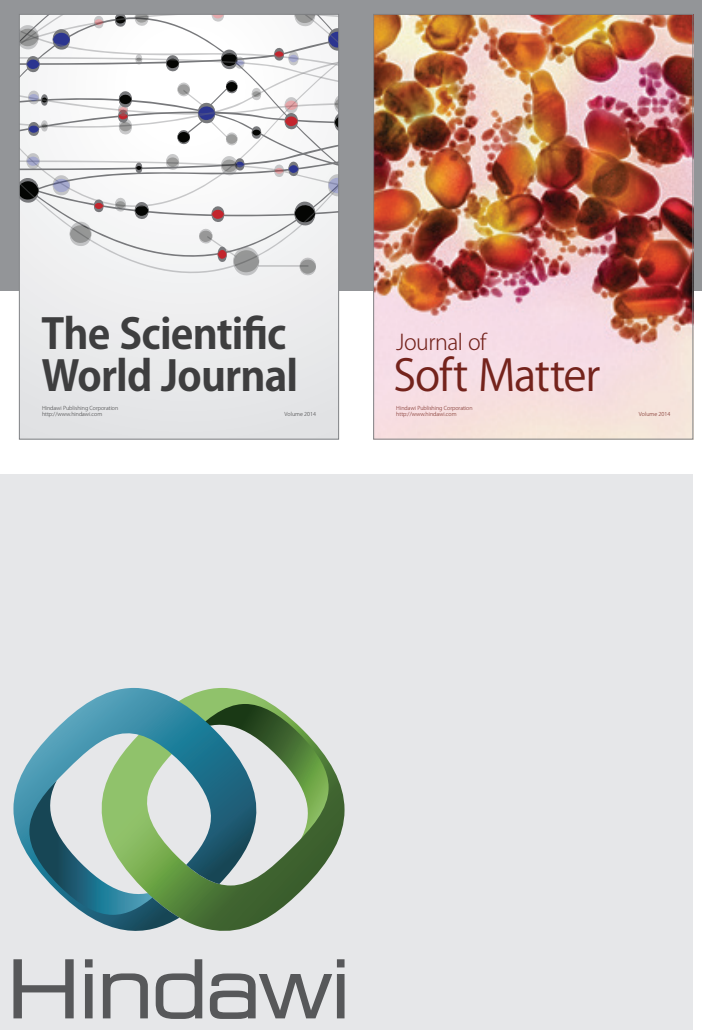

Submit your manuscripts at

http://www.hindawi.com

nternational Journal of

Statistical Mechanics
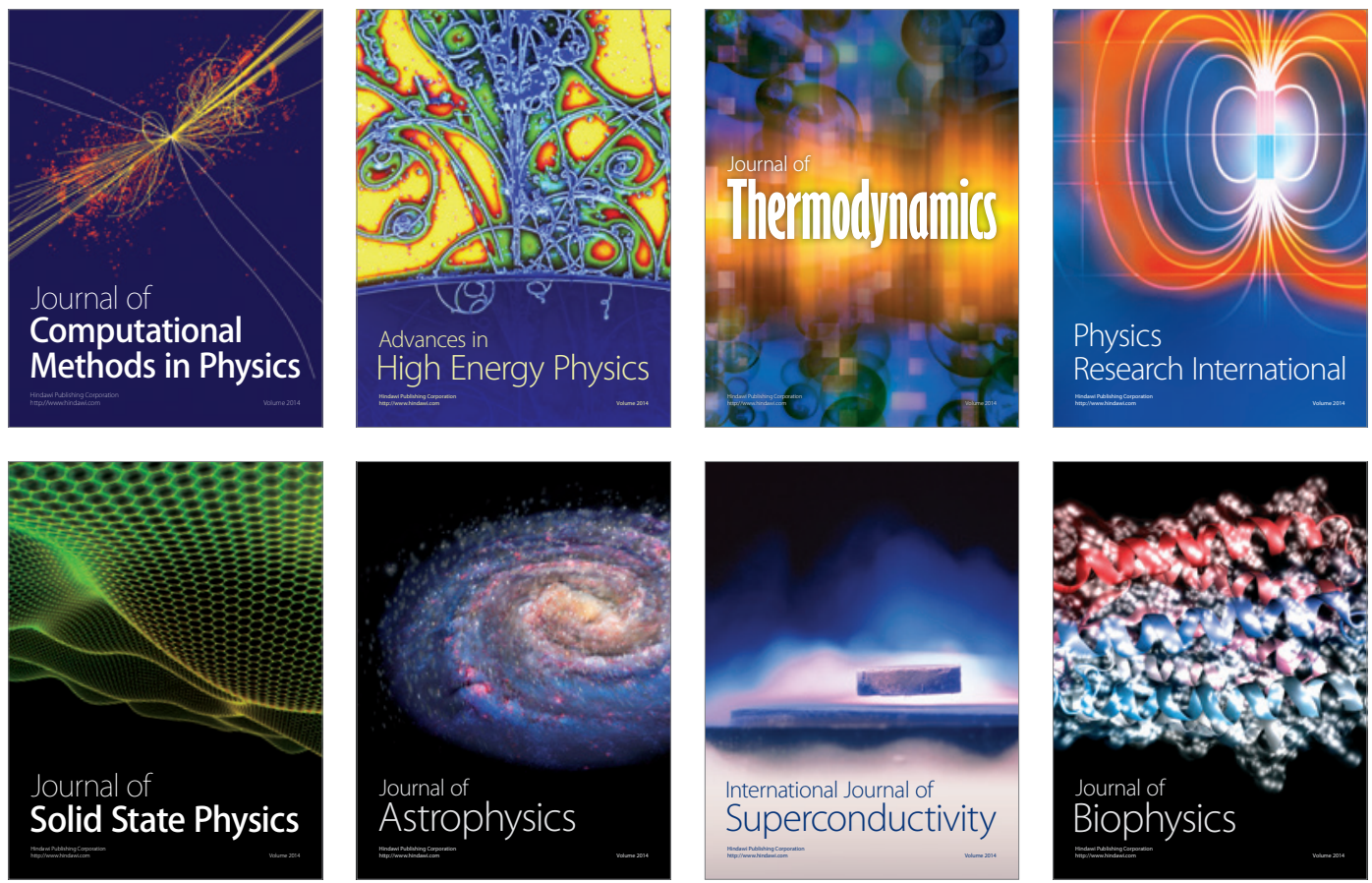
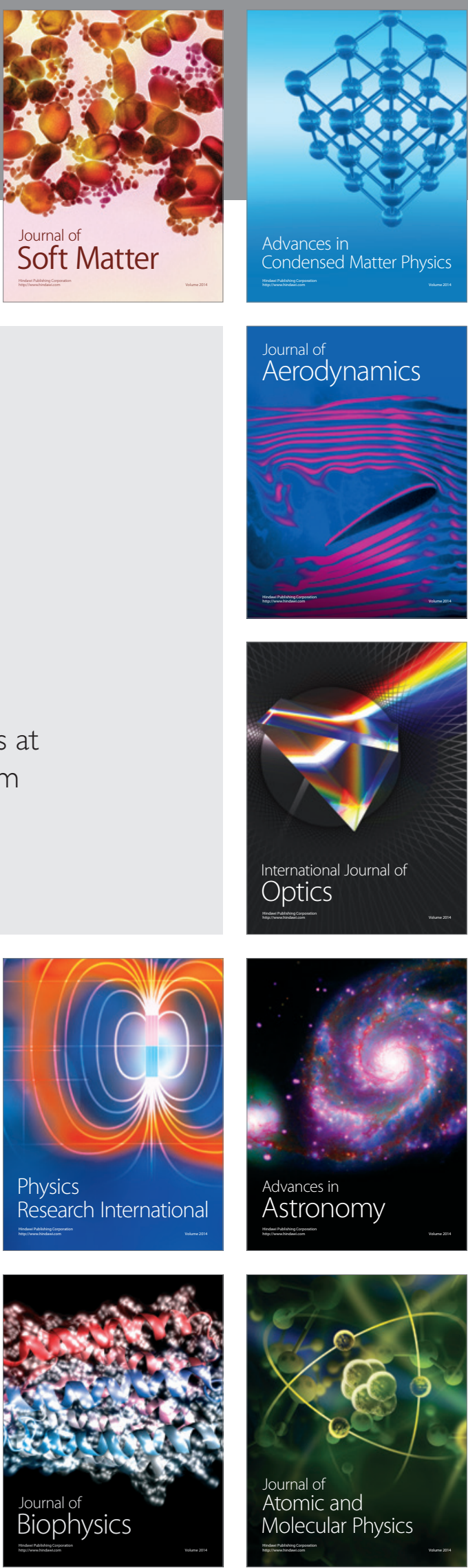